\title{
Fibrin-based factor delivery for therapeutic angiogenesis: friend or foe?
}

\author{
Ludovic Melly ${ }^{1,2} \cdot$ Andrea Banfi $^{1}$ (I)
}

Received: 15 October 2021 / Accepted: 7 February 2022 / Published online: 17 February 2022

(c) The Author(s) 2022

\begin{abstract}
Therapeutic angiogenesis aims at promoting the growth of blood vessels to restore perfusion in ischemic tissues or aid tissue regeneration. Vascular endothelial growth factor (VEGF) is the master regulator of angiogenesis in development, repair, and disease. However, exploiting VEGF for therapeutic purposes has been challenging and needs to take into account some key aspects of VEGF biology. In particular, the spatial localization of angiogenic signals within the extracellular matrix is crucial for physiological assembly and function of new blood vessels. Fibrin is the provisional matrix that is universally deposited immediately after injury and supports the initial steps of tissue regeneration. It provides therefore several ideal features as a substrate to promote therapeutic vascularization, especially through its ability to present growth factors in their physiological matrix-bound state and to modulate their availability for signaling. Here, we provide an overview of fibrin uses as a tissue-engineering scaffold material and as a tunable platform to finely control dose and duration of delivery of recombinant factors in therapeutic angiogenesis. However, in some cases, fibrin has also been associated with undesirable outcomes, namely the promotion of fibrosis and scar formation that actually prevent physiological tissue regeneration. Understanding the mechanisms that tip the balance between the pro- and anti-regenerative functions of fibrin will be the key to fully exploit its therapeutic potential.
\end{abstract}

Keywords Neovascularization $\cdot$ Vascular endothelial growth factor $\cdot$ Extracellular matrix $\cdot$ Ischemia $\cdot$ Fibrosis

\section{Therapeutic angiogenesis}

Ischemia is caused by inadequate balance between oxygen supply and demand. When happening in the heart, patients suffer from angina if the imbalance remains transient, or they undergo a myocardial infarction (MI) in case a complete occlusion occurs. Ischemic heart disease remains still the most common cause of death worldwide (Virani et al. 2021). Although angioplasty and surgery are available to restore the vascular flow distal to a stenosis, therapeutic angiogenesis can help as a concomitant therapy or even emerge as a standalone therapy if no other clinical intervention is feasible.

Ludovic Melly

ludovic.melly@uclouvain.be

Andrea Banfi

andrea.banfi@usb.ch

1 Department of Biomedicine, Basel University Hospital and University of Basel, Basel, Switzerland

2 Cardiac, Vascular and Thoracic Surgery Department, UCLouvain, CHU UCL Namur, Yvoir, Belgium
Therapeutic angiogenesis is broadly defined as a treatment strategy that promotes the growth of blood vessels to restore perfusion in ischemic tissues. Depending on where the process takes place a distinction should be made between angiogenesis and arteriogenesis. Angiogenesis is defined as the expansion of a tissue micro-vascular network starting from the pre-existing capillaries, which have a diameter of $8-12 \mu \mathrm{m}$ and are responsible for the metabolic exchanges. The most important physiological inducer of angiogenesis is ischemia. On the other hand, arteriogenesis refers to the formation of larger arterial vessels that channel the blood flow around the occlusion site as collaterals. This process instead consists of the enlargement and subsequent maturation of pre-existing small and poorly perfused collateral vessels that is induced mainly by shear stress and inflammatory cells but not directly by hypoxia. The two phenomena are related, since angiogenesis in downstream capillary beds directly stimulates upstream collateral remodeling and arteriogenesis (Annex 2013). The expansion of the micro-vascular bed, induced by angiogenic growth factors, is capable of causing the enlargement of upstream collateral arteries through both 
increased shear stress and gap junction-mediated retrograde signaling along vessel walls, thereby ensuring that blood perfusion downstream of the occlusion is restored by way of a biological bypass (Pries et al. 2010; Rissanen et al. 2005).

Therapeutic angiogenesis finds application in two distinct fields in regenerative medicine: (i) restoration of blood flow to ischemic tissue and (ii) rapid vascularization of tissueengineered grafts. In the first case, ischemia derives from a chronic or acute impairment of blood flow to tissue, leading to a loss of function and even necrosis. The most prevalent forms of ischemic disease are the result of progressive atherosclerosis with stenosis of major arteries in the lower limbs (peripheral artery disease or PAD) or in the heart (coronary artery disease or CAD). Diffuse microvascular impairment is an additional mechanism of tissue ischemia in type 2 diabetes, leading to non-healing cutaneous ulcers that are a major cause of morbidity in these patients (Virani et al. 2021). In these conditions, the promotion of vascular growth aims at restoring the blood supply to the chronically ischemic tissue that is still viable but dysfunctional, such as the hibernating myocardium in the border zone of an infarction.

On the other hand, tissue engineering aims at generating tissue replacements by combining suitable populations of progenitor cells with a biomaterial-based scaffold, with or without the addition of bioactive components, such as growth factors (Ahmed et al. 2008). Significant advances in stem cell biology and biomaterial engineering have made it possible to generate replacements of almost any tissue in the body. However, a major limiting factor for the clinical translation of this approach is the need for the rapid vascularization of the grafts upon in vivo implantation, to ensure the survival, differentiation, and function of the seeded progenitors inside the thick tissue-engineered constructs, since their clinically relevant size makes it impossible for oxygen and nutrients to reach deeper than about $1 \mathrm{~mm}$ by simple diffusion from surrounding vessels (Gianni-Barrera et al. 2020).

\section{Fundamental biological concepts in angiogenesis}

Vascular endothelial growth factor (VEGF) is the master regulator of angiogenesis, as this family of factors controls both physiological and pathological growth of blood and lymphatic vessels by signaling through their cognate receptors. The mammalian VEGF family comprises five main ligands (VEGF-A, -B, -C, and -D and placenta-derived growth factor, PlGF) and three receptors (VEGF-R1, -R2, and -R3). While the principal role of VEGF-C and -D is to stimulate lymphatic angiogenesis through VEGF-R3, blood vessel growth is mostly coordinated by the signaling of VEGF-A and -B and PIGF through R1 and R2.
VEGF is the most specific single factor capable of initiating the complex cascade of events leading to angiogenesis. Inactivation of VEGF during development results in embryonic lethality (Ferrara et al. 1996; Haigh et al. 2000), whereas VEGF delivery has been widely shown to induce new vascular growth in a variety of therapeutic models (YlaHerttuala et al. 2017). The best understood mechanism of angiogenesis is sprouting, which requires the formation of VEGF gradients in the microenvironment around each producing cell, thanks to its ability to bind to extracellular matrix (ECM) (Park et al. 1993). The orderly regulation of this morphogenic process requires endothelial cells to specialize into either migrating tip or proliferating stalk cells (Gerhardt et al. 2003) and is controlled by Notch signaling (Hellstrom et al. 2007). The first cells that respond to VEGF in the matrix become tip cells, which do not proliferate and are not involved in the formation of lumen structures, but rather sense the gradient of VEGF in the microenvironment and migrate towards it, thereby initiating the sprouting process. In response to VEGF, tip cells upregulate expression of Delta-like-4 (Dl14), which activates Notch-1 signaling on the neighboring endothelial cells and instructs them to acquire a stalk cell phenotype, instead. Contrary to tip cells, stalk cells respond to the total concentration of VEGF by proliferating rather than migrating and form new lumenized structures behind the sprouting tip.

However, sprouting is not the only process by which angiogenesis takes place. An alternative mechanism of vascular network expansion is intussusception, also referred to as splitting angiogenesis (Gianni-Barrera et al. 2014). Initially considered a kind of anatomical curiosity taking place only during the development of some organs, such as the lung and the kidney, intussusceptive angiogenesis has been increasingly recognized over the last decade as a common mechanism of vascular growth, with important therapeutic implications (Gianni-Barrera et al. 2020). Intussusception can be initiated very rapidly by increased blood flow and shear stress in the absence of growth factors (Egginton et al. 2001). However, very importantly, it is also the principal mechanism by which VEGF delivery at therapeutically relevant doses induces angiogenesis in clinically relevant tissues like skeletal muscle (Gianni-Barrera et al. 2013). How VEGF may induce sprouting or intussusception remains unclear, but its distribution in the matrix appears to be an important factor and the absence of a concentration gradient favors intussusception. This is suggested by comparing different outcomes of VEGF expression at different doses in the same tissue of skeletal muscle. In fact, spontaneous upregulation of VEGF at physiologically limited levels by ischemia leads to sprouting (Al Haj Zen et al. 2010), whereas its over-expression at significantly higher and therapeutic levels, which saturate the scarce matrix between muscle fibers and abrogate local gradients, induces angiogenesis by intussusception 
(Gianni-Barrera et al. 2013). During intussusceptive angiogenesis, endothelial cells respond to VEGF exclusively by proliferation without migration: no tip cells are formed and proliferating endothelium behaves functionally only as stalk cells. This leads to circumferential enlargement of the vessel, which then splits longitudinally into new vascular structures. Splitting requires the formation of endothelial pillars across the vascular lumen, which derive either from a vascular wall invagination that creates a contact between the opposite endothelial cells (Makanya et al. 2009), or by the extension and fusion of intraluminal filopodial-like protrusion from the endothelium (Egginton 2009). In both cases, subsequently, the endothelial junctions reorganize at the contact points and myofibroblast invade the core, stabilizing the structures into mature transluminal tissue pillars. Finally, these align longitudinally, fuse with each other, and split the affected vascular segment.

At the end of these morphogenic processes, newly formed endothelial structures are unstable and susceptible to rapid regression if VEGF signaling is interrupted. Vascular maturation and stabilization are the processes by which endothelial cells return to quiescence and independence from continued VEGF signaling and require the physical association of endothelium with a population of mesenchymal cells called pericytes. These are recruited by platelet-derived growth factor-BB (PDGF-BB), produced by activated endothelium, establish tight cell-to-cell contact with endothelial cells by residing under their basal lamina, and regulate their function through a complex cross-talk involving both paracrine and cell contact-dependent signaling pathways (Reginato et al. 2011). An active role in vascular maturation is also played by a recently identified population of myeloid cells (Giacca and Zacchigna 2012), which are recruited by Semaphorin-3a signaling through its receptor Neuropilin-1 and are therefore called neuropilin-expressing monocytes (NEM), and produce a host of pro-maturation paracrine factors, including PDGF-BB, Angiopoietin-1, and transforming growth factor- $\beta 1$ (TGF- $\beta 1$ ) (Groppa et al. 2015).

\section{Therapeutic requirements for angiogenic factor delivery}

During ischemia, hypoxic parenchymal cells are the main source of VEGF production, e.g., myofibers in skeletal muscle or cardiomyocytes in the heart (Banfi and GianniBarrera 2015; Braile et al. 2020). However, ischemiainduced upregulation of VEGF is tightly limited by the regulatory sequences in the endogenous promoter and this response may sometimes be insufficient to restore flow, necessitating delivery at supra-physiological levels in order to obtain a therapeutic effect. For example, a study of cellbased VEGF over-expression in ischemic muscle showed that functional restoration of blood flow required VEGF levels at least sixfold greater than those achieved by endogenous upregulation alone (von Degenfeld et al. 2006).

The selected aspects of the biology of vascular growth described above carry significant implications for the use of VEGF in therapeutic angiogenesis, particularly with respect to its dose, distribution in tissue, and duration of expression. In fact, it is well-known that uncontrolled over-expression of VEGF can induce severely aberrant vasculature, and even the growth of angioma-like vascular tumors in a variety of tissues, such as skeletal muscle (Ozawa et al. 2004; Springer et al. 1998), subcutaneous fat (Sundberg et al. 2001), the liver (Kitajima et al. 2005; Leppanen et al. 2006), and the heart (Lee et al. 2000; Schwarz et al. 2000). The discovery of VEGF sparked an immediate interest in its therapeutic potential and intense clinical investigation took place in the 1990s and 2000s to treat cardiovascular ischemia in both coronary and peripheral artery disease with VEGF delivery as a protein or by gene therapy vectors. However, after some promising initial reports, controlled clinical trials have generally failed to demonstrate therapeutic efficacy at safe vector doses, and clinical investigation of VEGF in cardiovascular disease has essentially stopped and reverted to preclinical experimentation. This first generation of cardiovascular VEGF clinical trials is discussed in several excellent reviews (Cooke and Losordo 2015; Rubanyi 2013). Later, VEGF protein delivery has also been investigated for the treatment of chronic foot ulcers in diabetic patients, and a product is clinically approved in some countries (telbermin), but efficacy is limited and approval has been revoked by the US FDA (Berry-Kilgour et al. 2021). A major issue limiting VEGF efficacy is that its therapeutic dosing, e.g., by gene delivery, has proven to be very challenging, with lower doses of gene therapy vectors being inefficacious and higher doses rapidly causing aberrant vascular growth (Rubanyi 2013). Subsequent studies revealed that this difficulty is related in part to one of the properties of VEGF that is crucial for its very biological function, namely its affinity for ECM. In fact, since VEGF remains tightly localized in the microenvironment around each producing cell after being secreted, different growth factor concentrations do not average with each other, even across distances of tens or hundredths of micrometers. Consequently, if expression is excessive in even just some areas, this is enough to cause aberrant vascular growth even if the total VEGF dose is rather low. However, if a homogeneous distribution of factor or of expression levels can be achieved, then controlled physiological angiogenesis is possible (Gianni-Barrera et al. 2020). This concept was clearly shown by studies using myoblast populations stably transduced with retroviral vectors to express VEGF in skeletal muscle (Ozawa et al. 2004). After retroviral transduction, different cells express a variety of levels due to differing viral copy numbers and their sites of chromatin 
integration. When the heterogenous transduced population was implanted, aberrant angioma-like structures were always induced, even if diluting it with non-expressing cells until very low total levels. However, by implanting monoclonal populations, derived from single cells isolated from the same heterogeneous population, in which every cell produced the same amount, it became clear that a wide range of VEGF levels exists that induce only normal, stable, and functional capillary networks and that angiomas are induced only by doses above a discrete threshold level. Furthermore, within the safe range, there exists a narrower therapeutic window, whereby too low doses efficiently induce normal angiogenesis but provide no therapeutic benefit, whereas higher ones induce the growth of normal vessels of larger caliber, which are also effective in forming collateral arteries and restoring blood flow in ischemic tissue (von Degenfeld et al. 2006). This concept is reproducible also without the tedious procedure of isolating monoclonal populations. FACS purification of transduced progenitors (e.g., bone marrow- or adiposederived mesenchymal stem cells), whose VEGF expression was suitably linked to a quantifiable cell surface marker, could rapidly generate populations producing defined and homogeneous VEGF levels (Helmrich et al. 2012). Implantation of such FACS-purified populations could avoid aberrant angiogenesis and efficiently induce only physiological and stable microvascular networks both in normal and chronically ischemic skeletal muscle (Misteli et al. 2010); (Wolff et al. 2012), as well as normal and ischemic myocardium (Melly et al. 2012, 2018).

The last key parameter to consider for therapeutically effective VEGF delivery is the duration of expression. In fact, although the development of new microvascular networks takes less than 7 days after VEGF gene delivery, the newly induced vessels are still unstable and, if VEGF signaling is lost before about 4 weeks, they can regress (Dor et al. 2002; Ozawa et al. 2004; Tafuro et al. 2009). Interestingly, the stabilization of VEGF-induced microvascular networks requires more than just pericyte recruitment, since new capillaries are already fully invested by normal pericytes 7 days after induction, but remains VEGF-dependent for a significantly longer period of time (Groppa et al. 2015). A specific population of Neuropilin1-expressing monocytes, recruited to sites of angiogenesis by Semaphorin3A secreted by activated endothelium, has been shown to play an important role in stabilizing new vessels after VEGF delivery (Groppa et al. 2015). The need to sustain VEGF delivery for about 4 weeks, but not indefinitely, is challenging for gene therapy approaches, since transient vectors (such as adenoviruses) provide expression for only about 7-10 days, whereas longer lasting vectors (such as adeno-associated and retro- or lentiviruses) do not switch off for months or even years, which raises safety concerns. The delivery of VEGF and other factors as recombinant proteins, rather than genetic information, is attractive to overcome the issues associated with heterogeneous levels in the tissue microenvironments, as a homogeneous dose distribution can be much more easily controlled (Martino et al. 2015). However, recombinant protein factors often suffer from too short half-lives in vivo. Their use in combination with biomaterials has the potential to exploit their potential and here we will specifically discuss the use of fibrin hydrogels for this purpose in regenerative medicine and therapeutic angiogenesis.

\section{Fibrin hydrogels for vascularization in regenerative medicine}

Fibrin is derived from the soluble precursor fibrinogen during the process of blood clotting and therefore is abundantly present in normal plasma. Fibrinogen is a large fibrous glycoprotein with three pairs of polypeptide chains. The fibrinopeptides, which are in the central region, are cleaved by thrombin to convert soluble fibrinogen to insoluble fibrin monomers, which then polymerize to make fibers and branch to yield a three-dimensional network with properties of a hydrogel - the fibrin clot (Weisel 2005). Since tissue damage involves in all cases hemorrhage and blood clotting, regeneration always starts with the deposition of a fibrinbased matrix rich in growth factors (Bao et al. 2009). In fact, the three polypeptide chains of fibrin contain multiple binding sites for growth factors, cellular receptors, and integrins, which make it an ideal substrate for cell adhesion and progenitor differentiation, as well as for endothelial migration and blood vessel in-growth (Petersen et al. 2018). Fibrin also provides very attractive features for therapeutic applications, as it is injectable as a liquid and it gels in situ without cytotoxicity, is remodeled by cell-associated enzymes (e.g. metalloproteinases, plasmin), providing a provisional matrix before the deposition of tissue-specific ECM, and can also be isolated from each patient to provide an autologous material (Breen et al. 2009). Therefore, fibrin has been extensively employed for tissue engineering approaches, and it is also investigated to a more limited extent as a delivery tool for growth factors in vivo. Beyond the works discussed here by way of conceptual examples, a more extensive listing detailing the current status of fibrin use in combination with growth factors for tissue regeneration in vivo can be found in a recent review by Anitua et al. (2019).

Tissue engineering is a strategy to produce biological substitutes for tissue lost due to surgery, trauma, or degeneration and it basically relies on the combination of tissuespecific progenitors/stem cells with suitable biomaterials that provide both $3 \mathrm{D}$ scaffolding and a biologically appropriate environment for progenitor proliferation and differentiation. These constructs also need to rapidly attract a vascular supply after in vivo implantation, in order to 
survive and produce mature tissue (Gianni-Barrera et al. 2020) (Fig. 1a). The properties of fibrin described above provide several desirable features for a tissue engineering scaffold and fibrin has been used successfully to regenerate a variety of tissues, including the adipose, cardiac and skeletal muscle, bone, cartilage, skin, and liver (Ahmed et al. 2008; Anitua et al. 2019). On the other hand, limitations of fibrin hydrogels as scaffolding material derive principally from its low mechanical stiffness and short duration in vivo, with degradation by invading cells within about a week that is in some cases insufficient for proper tissue formation by seeded progenitors. For this reason, the durability of fibrin hydrogels can be significantly extended to over 4 weeks by incorporating inhibitors of fibrinolysis, such as aprotinin, which allows a significant degree of control over the degradation kinetics of the hydrogels (Sacchi et al. 2014).

Fibrin exhibits a natural affinity for some growth factors, which is functional to its role in guiding the initial steps of regeneration after injury (Martino et al. 2013). However, in order to precisely control the regenerative microenvironment, as well as to exploit fibrin as a factor-delivery platform, a variety of technologies have been developed to incorporate specific doses and combinations of growth factors within fibrin hydrogels and to control their rate and mode of release (Fig. 1b).

Taking advantage of the natural process of fibrin formation itself, growth factors have been fused to a short octapeptide sequence that is the substrate of the transglutaminase coagulation factor XIIIa (TG-hook), allowing its covalent
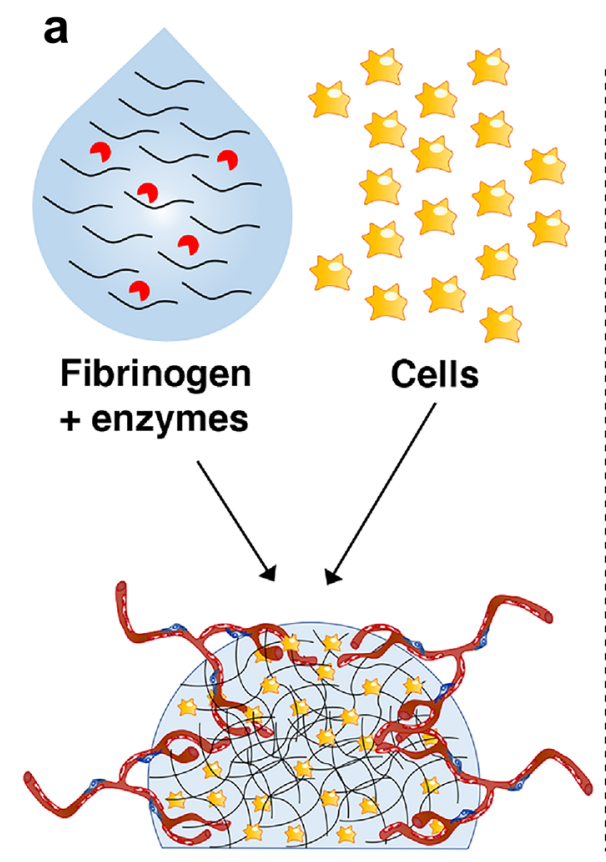

Fibrin scaffold

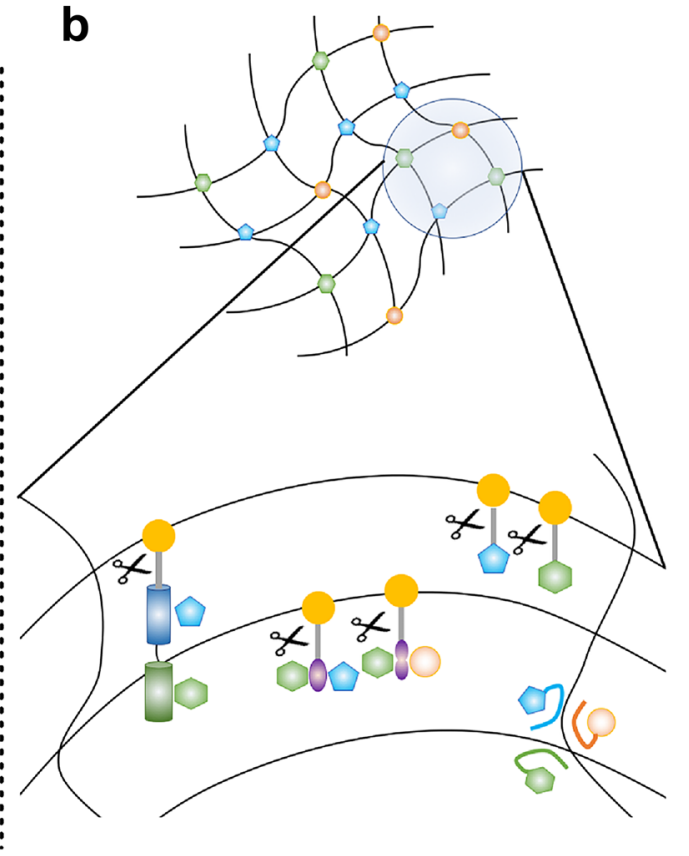

Factor-decorated fibrin matrices

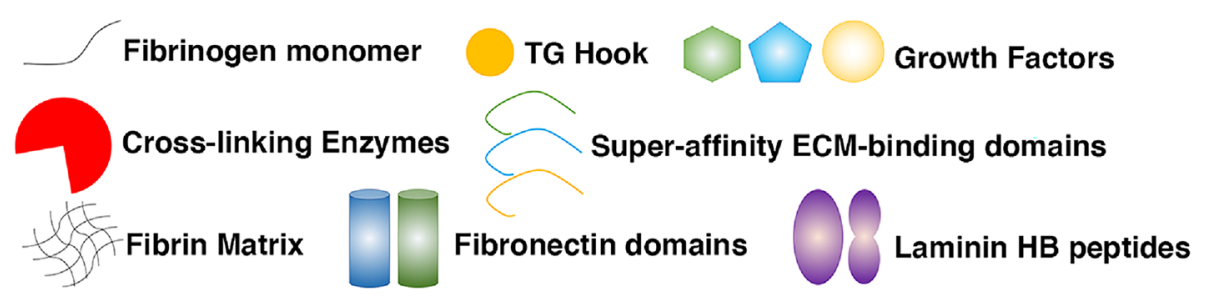

Fig. 1 Fibrin hydrogels in regenerative medicine. a Fibrin hydrogels provide a transient matrix for tissue engineered grafts that mimics the provisional matrix of physiological tissue regeneration, conducive to progenitor proliferation and differentiation, as well as to rapid invasion by host-derived blood vessels. b Several protein engineering approaches have been developed to decorate fibrin hydrogels with recombinant morphogens and growth factors. These approaches enable the use of fibrin hydrogels as tunable platforms for controlled release of factors in vivo to guide endogenous tissue repair, as well as to provide specific morphogenic microenvironments to seeded progenitors in tissue-engineered grafts 
cross-linking into fibrin hydrogels and release only by enzymatic cleavage (Zisch et al. 2001) (Ehrbar et al. 2004). By also including a TG-fused variant of the fibrinolysis inhibitor aprotinin (Lorentz et al. 2011), it was possible to independently control dose and duration of delivery in vivo of a growth factor like VEGF, developing fibrin hydrogels into a tunable factor release platform (Sacchi et al. 2014). In fact, for any given factor concentration in the hydrogel, the effective released dose per unit of time is directly proportional to the degradation rate of the matrix, while the duration of release is inversely proportional to the same parameter. Therefore, by fine-tuning TG-aprotinin concentrations, it was possible to sustain therapeutic delivery of a 500-fold dose range of VEGF in both normal and ischemic muscle over 4 weeks, as well as in a wound-healing model, with significant improvements in angiogenesis, tissue perfusion, and healing rate (Sacchi et al. 2014).

Different domains of ECM proteins have a promiscuous affinity for different growth factors. This property has been exploited to endow fibrin hydrogels with the ability to bind non-covalently, retain, and release gradually a variety of wild-type and endogenous factors, by cross-linking TG-hook-fused versions of specific domains of ECM proteins. Examples of this strategy include a multi-functional recombinant fragment of fibronectin, integrating both factorbinding and integrin-binding domains (Martino et al. 2011) and short sequences from the heparin-binding domains of the laminin $\alpha$-chain (Ishihara et al. 2018).

Alternatively, engineering of any growth factor with a peptide derived from placenta-derived growth factor-2 (PIGF-2) endows them with super-affinity for a broad range of ECM proteins, including fibrin, and also enables in vivo decoration of endogenous matrix with exogenously provided therapeutic proteins (Martino et al. 2014). The increased efficacy of the modified factors avoided the need to deliver supra-physiological doses, thereby also increasing safety, while effectively promoting diabetic wound healing and bone tissue repair.

\section{Fibrinogen-induced fibrosis - all is not so quiet on the fibrin front}

Alongside all the useful features described above, it has to be mentioned that, in some cases, fibrin can be associated with undesirable outcomes, particularly the promotion of fibrosis and scar formation that actually prevent physiological tissue regeneration. This phenomenon has been especially described in the central nervous system, where scar formation starts within hours after traumatic injury. Glial scar formation is a complex process, where important roles are played by at least three major cell populations: reactive astrocytes, NG2 glia, and microglia (Adams and Gallo 2018). Fibrinogen/fibrin that leaks into the CNS after vascular injury and disruption of the blood-brain barrier has pleiotropic effects on all these cells, both directly and indirectly (Fig. 2). For example, direct fibrin effects include activation of microglia by binding the $\mathrm{CD} 11 \mathrm{~b} / \mathrm{CD} 18$ integrin receptor, and the switch of NG2 glial progenitor differentiation from re-myelinating oligodendrocytes to reactive astrocytes, through activation of their activin A receptor type I (ACVR1) and BMP signaling (Petersen et al. 2018). Indirect actions of fibrin to promote glial scar formation have been identified in elegant work by Schachtrup and colleagues (Schachtrup et al. 2010). In fact, circulating fibrinogen is associated with latent TGF- $\beta$, which accumulates at the sites of vascular injury, is activated by interaction with astrocytes, and stimulates them to initiate scar formation via the Smad2 signaling pathway. This key role was shown by the fact that not only fibrinogen injection in the CNS was sufficient to induce scar formation in the absence of injury but also that genetic or pharmacologic depletion of fibrinogen, as well as inhibition of TGF- $\beta$ signaling, could abolish scar formation after injury. More recently, fibrin deposited after CNS injury has been shown to also stimulate neural stem cell differentiation to astrocytes over neurogenesis via BMP signaling (Pous et al. 2020).

Fibrin-induced fibrosis does not appear to be a phenomenon unique to the CNS. In fact, we recently found that fibrin hydrogels promote scar formation in the myocardium, while at the same time jeopardizing the therapeutic effect of angiogenic factor delivery (Melly et al. 2020). Fibrosis was not due to the presence of growth factors, tissue damage from the injection, the mechanical stiffness of the hydrogel or its volume, nor to an immune reaction. Rather, the presence of fibrin per se was enough to start the myocardial scarring process. Interestingly, despite the fact that fibrin hydrogels were completely degraded within 5 days, the resulting scars persisted at least 4 weeks without any sign of resolution, suggesting that, once started, the process is irreversible (Melly et al. 2020). Interestingly, a recent report by the group of Serena Zacchigna shows that treatment with a monoclonal antibody blocking a specific BMP1 isoform (BMP1.3) can reduce cardiac fibrosis and scar formation in models of myocardial infarction, in part through downregulation of TGF- $\beta$ signaling and myofibroblast activation (Vukicevic et al. 2022).

On the other hand, the usefulness of fibrin matrices for therapeutic factor delivery is established by solid evidence, as fibrin-induced fibrosis was not observed in a variety of other tissues and disease models (Anitua et al. 2019). For example, controlled VEGF delivery via the same fibrin-based platform in both normal and ischemic skeletal muscle, which is a contractile tissue like the myocardium or in ischemic skin wounds in mice, induced in all cases robust and functional angiogenesis without any sign of scar or fibrosis, restored blood flow and promoted healing (Sacchi et al. 2014). No skin fibrosis has ever been reported despite investigation of several fibrin-based matrices for growth factor delivery 
Fig. 2 The mechanisms of fibrinogen-induced CNS fibrosis. Fibrinogen can both directly and indirectly influence the function of several cell types involved in CNS fibrosis after vascular injury. Some of the best understood interactions are summarized here: (1) activation of microglia through binding of the integrin receptor CD11b/CD18; (2) differentiation of $\mathrm{NG} 2+$ progenitors to an astrocyte fate through activation of the activin A receptor type I (ACVR1) and BMP signaling; and (3) activation of latent TGF- $\beta$, bound to extravasated fibrinogen, which stimulates astrocytes to initiate matrix deposition and scar formation via Smad 2 signaling. LAP, latency-associated peptide; TGF-R1, TGF $\beta$-receptor 1

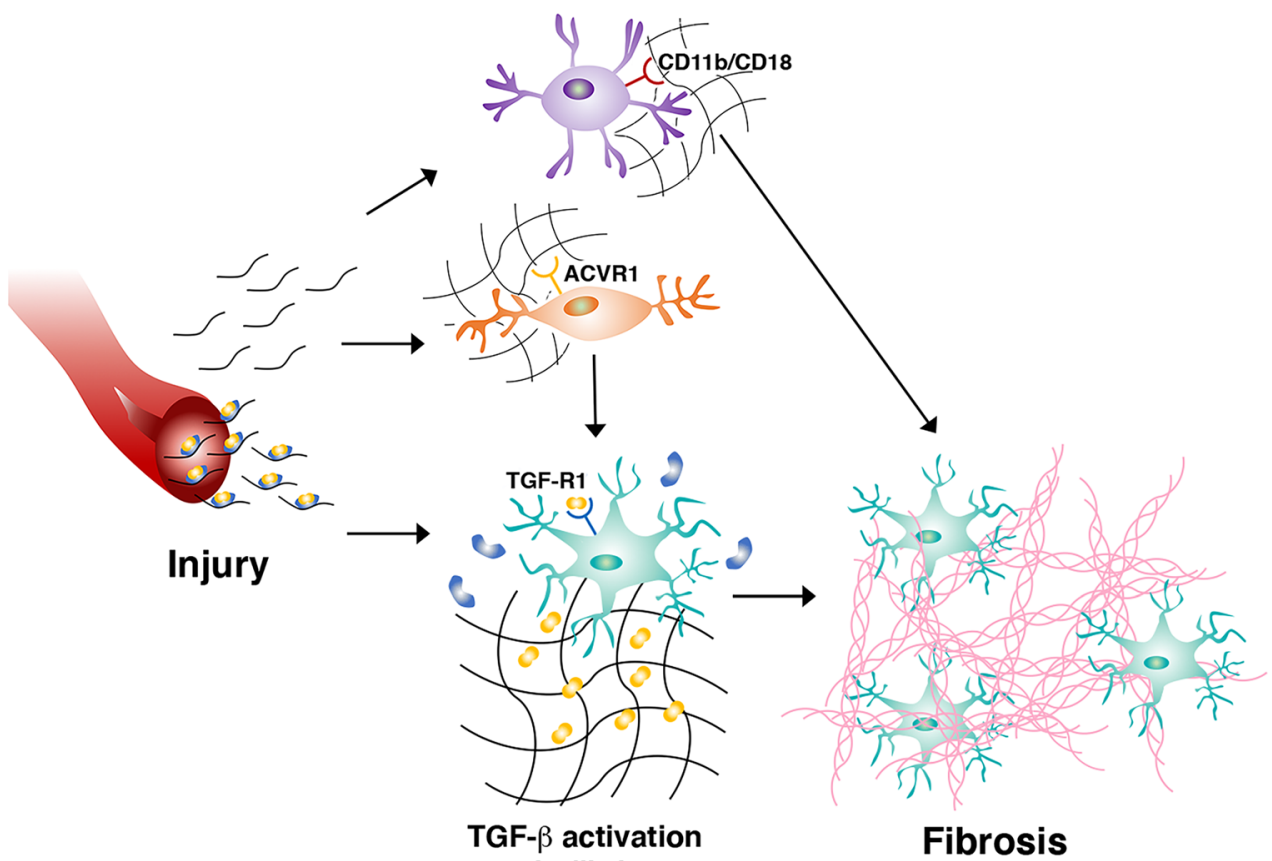

in fibrin

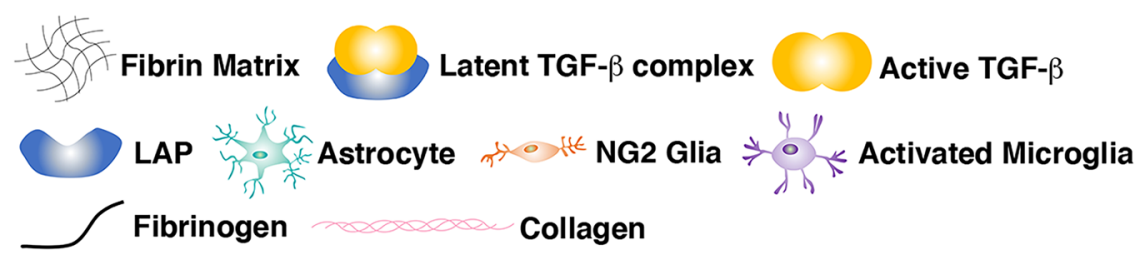

in diabetic wound healing (Whelan et al. 2014). Bladder smooth muscle has also been targeted with a bioactive fibrinbased bulking material to treat urinary incontinence, without any report of ensuing fibrosis (Vardar et al. 2019).

\section{Conclusions}

Fibrin is the natural matrix that provides the first environment for the initiation of all forms of repair after injury. Not surprisingly, it provides attractive biological conditions for the therapeutic delivery of progenitor cells and morphogens in many fields of regenerative medicine. The specific combinations and doses of factors and duration of delivery will likely depend on the specific tissue and clinical application, as well as the possible inclusion of vascular cells in the matrix to provide more rapid vascularization and paracrine factors. For example, chronic skin wounds in diabetic patients present rather different features than large volumes of ischemic muscle in peripheral artery disease, and these are expected to require tailored therapeutic approaches. The flexible tools afforded by fibrin-based delivery platforms can be very useful to investigate the individual therapeutic needs. However, in some conditions, fibrin can also activate anti-regenerative processes, stimulating fibrosis and scar formation, with impairment of angiogenesis and stem cell differentiation. What mechanisms may tip the balance and specify one outcome or the other is still poorly understood, despite recent advances. Elucidating the molecular underpinning of fibrin-induced fibrosis will be paramount to overcome the scarring process and enable the exploitation of this attractive therapeutic platform for regenerative medicine.

Acknowledgements We are grateful to Dr. Nunzia Di Maggio (Basel University Hospital) for the help with figure preparation.

Funding Open access funding provided by University of Basel. This work was supported by grants by the Swiss National Science Foundation (182357), the Swiss Nanoscience Institute (Nano-Argovia project KOKORO) and the European Union H2020 Program (801159 and 874790 ) to A.B., as well as an intramural grant of the CHU UCL Namur (Mont Godinne Foundation) to L.M.

\section{Declarations}

Ethics approval This article does not contain any studies with human participants or animals performed by any of the authors.

Conflict of interest The authors declare no competing interests.

Open Access This article is licensed under a Creative Commons Attribution 4.0 International License, which permits use, sharing, adaptation, distribution and reproduction in any medium or format, as long 
as you give appropriate credit to the original author(s) and the source, provide a link to the Creative Commons licence, and indicate if changes were made. The images or other third party material in this article are included in the article's Creative Commons licence, unless indicated otherwise in a credit line to the material. If material is not included in the article's Creative Commons licence and your intended use is not permitted by statutory regulation or exceeds the permitted use, you will need to obtain permission directly from the copyright holder. To view a copy of this licence, visit http://creativecommons.org/licenses/by/4.0/.

\section{References}

Adams KL, Gallo V (2018) The diversity and disparity of the glial scar. Nat Neurosci 21(1):9-15. https://doi.org/10.1038/s41593-017-0033-9

Ahmed TA, Dare EV, Hincke M (2008) Fibrin: a versatile scaffold for tissue engineering applications. Tissue Eng Part B Rev 14(2):199-215. https://doi.org/10.1089/ten.teb.2007.0435

Anitua E, Nurden P, Prado R, Nurden AT, Padilla S (2019) Autologous fibrin scaffolds: when platelet- and plasma-derived biomolecules meet fibrin. Biomaterials 192:440-460. https://doi. org/10.1016/j.biomaterials.2018.11.029

Annex BH (2013) Therapeutic angiogenesis for critical limb ischaemia. Nat Rev Cardiol 10(7):387-396. https://doi.org/10.1038/ nrcardio. 2013.70

Banfi A, Gianni-Barrera R (2015) VEGF, shear stress and muscle angiogenesis: a complicated triangle. Acta Physiol (oxf) 214(3):298-299. https://doi.org/10.1111/apha.12514

Bao P, Kodra A, Tomic-Canic M, Golinko MS, Ehrlich HP, Brem H (2009) The role of vascular endothelial growth factor in wound healing. J Surg Res 153(2):347-358. https://doi.org/10.1016/j. jss.2008.04.023

Berry-Kilgour C, Cabral J, Wise L (2021) Advancements in the delivery of growth factors and cytokines for the treatment of cutaneous wound indications. Adv Wound Care 10(11):596622. https://doi.org/10.1089/wound.2020.1183

Braile M, Marcella S, Cristinziano L, Galdiero MR, Modestino L, Ferrara AL, Loffredo S (2020) VEGF-A in cardiomyocytes and heart diseases. Int J Mol Sci 21(15). https://doi.org/10.3390/ ijms 21155294

Breen A, O'Brien T, Pandit A (2009) Fibrin as a delivery system for therapeutic drugs and biomolecules. Tissue Eng Part B Rev 15(2):201-214. https://doi.org/10.1089/ten.TEB.2008.0527

Cooke JP, Losordo DW (2015) Modulating the vascular response to limb ischemia: angiogenic and cell therapies. Circ Res 116(9):15611578. https://doi.org/10.1161/CIRCRESAHA.115.303565

Dor Y, Djonov V, Abramovitch R, Itin A, Fishman GI, Carmeliet P, Keshet E (2002) Conditional switching of VEGF provides new insights into adult neovascularization and pro-angiogenic therapy. EMBO J 21(8):1939-1947. https://doi.org/10.1093/ emboj/21.8.1939

Egginton S (2009) Invited review: activity-induced angiogenesis. Pflugers Arch 457(5):963-977. https://doi.org/10.1007/ s00424-008-0563-9

Egginton S, Zhou AL, Brown MD, Hudlicka O (2001) Unorthodox angiogenesis in skeletal muscle. Cardiovasc Res 49(3):634-646. https://doi.org/10.1016/s0008-6363(00)00282-0

Ehrbar M, Djonov VG, Schnell C, Tschanz SA, Martiny-Baron G, Schenk U, Zisch AH (2004) Cell-demanded liberation of VEGF121 from fibrin implants induces local and controlled blood vessel growth. Circ Res 94(8):1124-1132. https://doi.org/ 10.1161/01.RES.0000126411.29641.08

Ferrara N, Carver-Moore K, Chen H, Dowd M, Lu L, O'Shea KS, Moore MW (1996) Heterozygous embryonic lethality induced by targeted inactivation of the VEGF gene. Nature 380(6573):439-442. https://doi.org/10.1038/380439a0
Gerhardt H, Golding M, Fruttiger M, Ruhrberg C, Lundkvist A, Abramsson A, Betsholtz C (2003) VEGF guides angiogenic sprouting utilizing endothelial tip cell filopodia. J Cell Biol 161(6):1163-1177. https://doi.org/10.1083/jcb.200302047

Giacca M, Zacchigna S (2012) VEGF gene therapy: therapeutic angiogenesis in the clinic and beyond. Gene Ther 19(6):622-629. https://doi.org/10.1038/gt.2012.17

Gianni-Barrera R, Bartolomeo M, Vollmar B, Djonov V, Banfi A (2014) Split for the cure: VEGF, PDGF-BB and intussusception in therapeutic angiogenesis. Biochem Soc Trans 42(6):16371642. https://doi.org/10.1042/BST20140234

Gianni-Barrera R, Di Maggio N, Melly L, Burger MG, Mujagic E, Gurke L, Banfi A (2020) Therapeutic vascularization in regenerative medicine: concise review. Stem Cells Transl Med. https:// doi.org/10.1002/sctm.19-0319

Gianni-Barrera R, Trani M, Fontanellaz C, Heberer M, Djonov V, Hlushchuk R, Banfi A (2013) VEGF over-expression in skeletal muscle induces angiogenesis by intussusception rather than sprouting. Angiogenesis 16(1):123-136. https://doi.org/ 10.1007/s10456-012-9304-y

Groppa E, Brkic S, Bovo E, Reginato S, Sacchi V, Di Maggio N, Banfi A (2015) VEGF dose regulates vascular stabilization through Semaphorin3A and the Neuropilin-1+ monocyte/TGFbeta1 paracrine axis. EMBO Mol Med 7(10):1366-1384. https:// doi.org/10.15252/emmm.201405003

Haigh JJ, Gerber HP, Ferrara N, Wagner EF (2000) Conditional inactivation of VEGF-A in areas of collagen2a1 expression results in embryonic lethality in the heterozygous state. Development 127(7):1445-1453. Retrieved from https://www.ncbi.nlm.nih. gov/pubmed/10704390

Hellstrom M, Phng LK, Hofmann JJ, Wallgard E, Coultas L, Lindblom P, Betsholtz C (2007) Dll4 signalling through Notch1 regulates formation of tip cells during angiogenesis. Nature 445(7129):776780. https://doi.org/10.1038/nature05571

Helmrich U, Marsano A, Melly L, Wolff T, Christ L, Heberer M, Banfi A (2012) Generation of human adult mesenchymal stro$\mathrm{mal} / \mathrm{stem}$ cells expressing defined xenogenic vascular endothelial growth factor levels by optimized transduction and flow cytometry purification. Tissue Eng Part C Methods 18(4):283292. https://doi.org/10.1089/ten.TEC.2011.0413

Ishihara J, Ishihara A, Fukunaga K, Sasaki K, White MJV, Briquez PS, Hubbell JA (2018) Laminin heparin-binding peptides bind to several growth factors and enhance diabetic wound healing. Nat Commun 9(1):2163. https://doi.org/10.1038/s41467-018-04525-w

Kitajima S, Liu E, Morimoto M, Koike T, Yu Y, Watanabe T, Fan J (2005) Transgenic rabbits with increased VEGF expression develop hemangiomas in the liver: a new model for KasabachMerritt syndrome. Lab Invest 85(12):1517-1527. https://doi.org/ 10.1038/labinvest.3700346

Lee RJ, Springer ML, Blanco-Bose WE, Shaw R, Ursell PC, Blau HM (2000) VEGF gene delivery to myocardium: deleterious effects of unregulated expression. Circulation 102(8):898-901. https://doi. org/10.1161/01.cir.102.8.898

Leppanen P, Kholova I, Mahonen AJ, Airenne K, Koota S, Mansukoski H, Yla-Herttuala S (2006) Short and long-term effects of hVEGF$\mathrm{A}(165)$ in Cre-activated transgenic mice. PLoS ONE 1:e13. https:// doi.org/10.1371/journal.pone.0000013

Lorentz KM, Kontos S, Frey P, Hubbell JA (2011) Engineered aprotinin for improved stability of fibrin biomaterials. Biomaterials 32(2):430-438. https://doi.org/10.1016/j.biomaterials.2010.08.109

Makanya AN, Hlushchuk R, Djonov VG (2009) Intussusceptive angiogenesis and its role in vascular morphogenesis, patterning, and remodeling. Angiogenesis 12(2):113-123. https://doi.org/10. 1007/s10456-009-9129-5

Martino MM, Briquez PS, Guc E, Tortelli F, Kilarski WW, Metzger S, Hubbell JA (2014) Growth factors engineered for super-affinity to the 
extracellular matrix enhance tissue healing. Science 343(6173):885888. https://doi.org/10.1126/science.1247663

Martino MM, Briquez PS, Ranga A, Lutolf MP, Hubbell JA (2013) Heparin-binding domain of fibrin(ogen) binds growth factors and promotes tissue repair when incorporated within a synthetic matrix. Proc Natl Acad Sci U S A 110(12):4563-4568. https:// doi.org/10.1073/pnas.1221602110

Martino MM, Brkic S, Bovo E, Burger M, Schaefer DJ, Wolff T, Banfi A (2015) Extracellular matrix and growth factor engineering for controlled angiogenesis in regenerative medicine. Front Bioeng Biotechnol 3:45. https://doi.org/10.3389/fbioe. 2015.00045

Martino MM, Tortelli F, Mochizuki M, Traub S, Ben-David D, Kuhn GA, Hubbell JA (2011) Engineering the growth factor microenvironment with fibronectin domains to promote wound and bone tissue healing. Sci Transl Med 3(100):100ra189. https:// doi.org/10.1126/scitranslmed.3002614

Melly L, Boccardo S, Eckstein F, Banfi A, Marsano A (2012) Cell and gene therapy approaches for cardiac vascularization. Cells 1(4):961-975. https://doi.org/10.3390/cells1040961

Melly L, Cerino G, Frobert A, Cook S, Giraud MN, Carrel T, Banfi A (2018) Myocardial infarction stabilization by cell-based expression of controlled vascular endothelial growth factor levels. J Cell Mol Med 22(5):2580-2591. https://doi.org/10.1111/jcmm.13511

Melly L, Grosso A, Stanciu Pop C, Yu-Hsuan C, Nollevaux MC, Schachtrup C, Banfi A (2020) Fibrin hydrogels promote scar formation and prevent therapeutic angiogenesis in the heart. $\mathrm{J}$ Tissue Eng Regen Med 14(10):1513-1523. https://doi.org/10. 1002/term.3118

Misteli H, Wolff T, Fuglistaler P, Gianni-Barrera R, Gurke L, Heberer M, Banfi A (2010) High-throughput flow cytometry purification of transduced progenitors expressing defined levels of vascular endothelial growth factor induces controlled angiogenesis in vivo. Stem Cells 28(3):611-619. https://doi.org/10. 1002/stem.291

Ozawa CR, Banfi A, Glazer NL, Thurston G, Springer ML, Kraft PE, Blau HM (2004) Microenvironmental VEGF concentration, not total dose, determines a threshold between normal and aberrant angiogenesis. J Clin Invest 113(4):516-527. https://doi.org/10. 1172/JCI18420

Park JE, Keller GA, Ferrara N (1993) The vascular endothelial growth factor (VEGF) isoforms: differential deposition into the subepithelial extracellular matrix and bioactivity of extracellular matrix-bound VEGF. Mol Biol Cell 4(12):1317-1326. https://doi.org/10.1091/mbc.4.12.1317

Petersen MA, Ryu JK, Akassoglou K (2018) Fibrinogen in neurological diseases: mechanisms, imaging and therapeutics. Nat Rev Neurosci 19(5):283-301. https://doi.org/10.1038/nrn.2018.13

Pous L, Deshpande SS, Nath S, Mezey S, Malik SC, Schildge S, Schachtrup C (2020) Fibrinogen induces neural stem cell differentiation into astrocytes in the subventricular zone via BMP signaling. Nat Commun 11(1):630. https://doi.org/10.1038/ s41467-020-14466-y

Pries AR, Hopfner M, le Noble F, Dewhirst MW, Secomb TW (2010) The shunt problem: control of functional shunting in normal and tumour vasculature. Nat Rev Cancer 10(8):587-593. https://doi. org/10.1038/nrc2895

Reginato S, Gianni-Barrera R, Banfi A (2011) Taming of the wild vessel: promoting vessel stabilization for safe therapeutic angiogenesis. Biochem Soc Trans 39(6):1654-1658. https://doi.org/ 10.1042/BST20110652

Rissanen TT, Korpisalo P, Markkanen JE, Liimatainen T, Orden MR, Kholova I, Yla-Herttuala S (2005) Blood flow remodels growing vasculature during vascular endothelial growth factor gene therapy and determines between capillary arterialization and sprouting angiogenesis. Circulation 112(25):3937-3946. https:// doi.org/10.1161/CIRCULATIONAHA.105.543124

Rubanyi GM (2013) Mechanistic, technical, and clinical perspectives in therapeutic stimulation of coronary collateral development by angiogenic growth factors. Mol Ther 21(4):725-738. https:// doi.org/10.1038/mt.2013.13

Sacchi V, Mittermayr R, Hartinger J, Martino MM, Lorentz KM, Wolbank S, Banfi A (2014) Long-lasting fibrin matrices ensure stable and functional angiogenesis by highly tunable, sustained delivery of recombinant VEGF164. Proc Natl Acad Sci U S A 111(19):6952-6957. https://doi.org/10.1073/pnas.1404605111

Schachtrup C, Ryu JK, Helmrick MJ, Vagena E, Galanakis DK, Degen JL, Akassoglou K (2010) Fibrinogen triggers astrocyte scar formation by promoting the availability of active TGF-beta after vascular damage. J Neurosci 30(17):5843-5854. https:// doi.org/10.1523/JNEUROSCI.0137-10.2010

Schwarz ER, Speakman MT, Patterson M, Hale SS, Isner JM, Kedes LH, Kloner RA (2000) Evaluation of the effects of intramyocardial injection of DNA expressing vascular endothelial growth factor (VEGF) in a myocardial infarction model in the rat-angiogenesis and angioma formation. J Am Coll Cardiol 35(5):1323-1330. https://doi.org/10.1016/s0735-1097(00)00522-2

Springer ML, Chen AS, Kraft PE, Bednarski M, Blau HM (1998) VEGF gene delivery to muscle: potential role for vasculogenesis in adults. Mol Cell 2(5):549-558. Retrieved from http://www. ncbi.nlm.nih.gov/pubmed/9844628

Sundberg C, Nagy JA, Brown LF, Feng D, Eckelhoefer IA, Manseau EJ, Dvorak HF (2001) Glomeruloid microvascular proliferation follows adenoviral vascular permeability factor/vascular endothelial growth factor-164 gene delivery. Am J Pathol 158(3):11451160. https://doi.org/10.1016/S0002-9440(10)64062-X

Tafuro S, Ayuso E, Zacchigna S, Zentilin L, Moimas S, Dore F, Giacca M (2009) Inducible adeno-associated virus vectors promote functional angiogenesis in adult organisms via regulated vascular endothelial growth factor expression. Cardiovasc Res 83(4):663-671. https://doi.org/10.1093/cvr/cvp152

Vardar E, Vythilingam G, Pinnagoda K, Engelhardt EM, Zambelli PY, Hubbell JA, Larsson HM (2019) A bioactive injectable bulking material; a potential therapeutic approach for stress urinary incontinence. Biomaterials 206:41-48. https://doi.org/ 10.1016/j.biomaterials.2019.03.030

Virani SS, Alonso A, Aparicio HJ, Benjamin EJ, Bittencourt MS, Callaway CW (2021) Heart Disease and Stroke Statistics2021 update: a report From the American Heart Association. Circulation 143(8):e254-e743. https://doi.org/10.1161/CIR. 0000000000000950

von Degenfeld G, Banfi A, Springer ML, Wagner RA, Jacobi J, Ozawa CR, Blau HM (2006) Microenvironmental VEGF distribution is critical for stable and functional vessel growth in ischemia. FASEB J 20(14):2657-2659. https://doi.org/10.1096/ fj.06-6568fje

Vukicevic S, Colliva A, Kufner V, Martinelli V, Moimas S, Vodret S, Zacchigna S (2022) Bone morphogenetic protein 1.3 inhibition decreases scar formation and supports cardiomyocyte survival after myocardial infarction. Nat Commun 13(1):81. https://doi. org/10.1038/s41467-021-27622-9

Weisel JW (2005) Fibrinogen and fibrin. Adv Protein Chem 70:247299. https://doi.org/10.1016/S0065-3233(05)70008-5

Whelan D, Caplice NM, Clover AJ (2014) Fibrin as a delivery system in wound healing tissue engineering applications. J Control Release 196:1-8. https://doi.org/10.1016/j.jconrel.2014.09.023

Wolff T, Mujagic E, Gianni-Barrera R, Fueglistaler P, Helmrich U, Misteli H, Banfi A (2012) FACS-purified myoblasts producing controlled VEGF levels induce safe and stable angiogenesis in chronic hind limb ischemia. J Cell Mol Med 16(1):107-117. https://doi.org/10.1111/j.1582-4934.2011.01308.x 
Yla-Herttuala S, Bridges C, Katz MG, Korpisalo P (2017) Angiogenic gene therapy in cardiovascular diseases: dream or vision? Eur Heart J 38(18):1365-1371. https://doi.org/10.1093/eurheartj/ ehw547

Zen AHA, Oikawa A, Bazan-Peregrino M, Meloni M, Emanueli C, Madeddu, P (2010) Inhibition of delta-like-4-mediated signaling impairs reparative angiogenesis after ischemia. Circ Res 107(2):283-293. https://doi.org/10.1161/CIRCRESAHA.110. 221663
Zisch AH, Schenk U, Schense JC, Sakiyama-Elbert SE, Hubbell JA (2001) Covalently conjugated VEGF-fibrin matrices for endothelialization. J Control Release 72(1-3):101-113. https://doi.org/10. $1016 / \mathrm{s} 0168-3659(01) 00266-8$

Publisher's Note Springer Nature remains neutral with regard to jurisdictional claims in published maps and institutional affiliations. 